\title{
Rotavirus-Like Particles: A Novel Nanocarrier for the Gut
}

\author{
Naima G. Cortes-Perez, ${ }^{1,2,3}$ Catherine Sapin, ${ }^{1,2}$ Loïc Jaffrelo,, , 2 Sabine Daou,, 2 \\ Jean Pierre Grill,, ${ }^{1,2}$ Philippe Langella, ${ }^{3}$ Philippe Seksik, ${ }^{1,2,4}$ Laurent Beaugerie, , 2, 4 \\ Serge Chwetzoff, ${ }^{1,2,5}$ and Germain Trugnan ${ }^{1,2}$ \\ ${ }^{1}$ UPMC University Paris 06, UMRS 538 “Trafic membranaire et signalisation dans les cellules épithéliales”, 27 rue de Chaligny, \\ 75012 Paris, France \\ ${ }^{2}$ INSERM UMRS 538 “Trafic membranaire et signalisation dans les cellules épithéliales”, CHU Saint Antoine, 27 rue de Chaligny, \\ 75012 Paris, France \\ ${ }^{3}$ Unité INRA d'Ecologie et de Physiologie du Système Digestif, Domaine de Vilvert, Jouy-en-Josas, France \\ ${ }^{4}$ AP-HP, Hôpital Saint-Antoine, Service de Gastroentérologie et Nutrition, 184 rue du Faubourg Saint Antoine, 75012 Paris, France \\ ${ }^{5}$ Unité de Virologie et Immunologie Moléculaires, UR 089 INRA, 78352 Jouy-en-Josas, France \\ Correspondence should be addressed to Germain Trugnan, germain.trugnan@upmc.fr
}

Received 19 July 2009; Revised 23 November 2009; Accepted 4 February 2010

Academic Editor: Jianguo Wu

Copyright () 2010 Naima G. Cortes-Perez et al. This is an open access article distributed under the Creative Commons Attribution License, which permits unrestricted use, distribution, and reproduction in any medium, provided the original work is properly cited.

\begin{abstract}
The delivery of bioactive molecules directly to damaged tissues represents a technological challenge. We propose here a new system based on virus-like particles (VLP) from rotavirus, with a marked tropism for the gut to deliver bio-active molecules to intestinal cells. For this, nonreplicative VLP nanoparticles were constructed using a baculovirus expression system and used to deliver an exogenous biomolecule, the green fluorescent protein (GFP), into either MA104 cells or intestinal cells from healthy and 2,4,6trinitrobenzene sulfonic acid (TNBS)-treated mice. Our results show that expression of rotavirus capsid proteins in baculovirus led to the auto assembly of VLP that display similar properties to rotavirus. In vitro experiments showed that VLP were able to enter into MA104 cells and deliver the reporter protein. Intragastric administration of fluorescent VLP in healthy and TNBStreated mice resulted in the detection of GFP and viral proteins in intestinal samples. Our results demonstrate an efficient entry of non-replicative rotavirus VLP into the epithelial cell line MA104 and provide the first in vivo evidence of the potential of these nanoparticles as a promising safe candidate for drug delivery to intestinal cells.
\end{abstract}

\section{Introduction}

Delivery systems able to efficiently transfer bioactive molecules to specific target tissue represent a technological challenge. Viral vectors are under intensive investigation as efficient delivery systems to be used in clinical trials because of their natural invasive characteristics and tropism $[1,2]$. However, viral vectors lack specificity for intestinal damaged tissues and could display replicative properties that may elicit side-effects [3]. In this context, an attractive strategy is the elimination of the genetic material in order to turn them into replication-defective vectors, and use only the empty particles as nanoboxes for biomolecule delivery. Rotaviruses, members of the Reoviridae family, exhibit a marked tropism for the intestinal epithelium. Their capsid consists of three concentric layers (i) the outer-most layer, which is composed of VP7 and VP4 proteins; (ii) the inner layer composed of trimers of VP6 protein, and (iii) the core which is mostly composed of a nucleic acid binding protein, VP2. Coexpression of capsid viral proteins in the baculovirus expression system leads to the production of nonreplicative rotavirus derived virus-like particles (VLP) [4]. Several studies have demonstrated that VLP display properties very similar to those of rotavirus and that they lack infectivity [5-8]. Thus, their natural tropism and nonreplicative properties make rotavirus-derived VLP a promising safe candidate for drug delivery to intestine in pathologies such as inflammatory bowel diseases (IBD). We hypothesized that this vector must be able to deliver in situ (in pathological tissues) therapeutics molecules as anti-inflammatory proteins or RNAi to interfere 
with the proinflammatory pathway of $\mathrm{NF} \kappa \mathrm{B}$, using the same mechanism used by Rotavirus to infect and deliver the RNA to intestinal cells. It has been reported that heterologous proteins as green fluorescent protein (GFP) could genetically fused to VP2 and incorporated into VLP [4]. In order to assure the correct VLP assembly, we consider that molecules smaller than of $\sim 250$ amino acids could easily be integrated into this delivery system by a fusion to VP2 protein. In the present work, we used home-made fluorescent VLP (VLPGFP), previously reported by Charpilienne et al. [4], which are composed by the fusion of green fluorescent protein (GFP) to VP2 protein (GFP:VP2). We controlled their quality and we evaluated their potential as delivery a system to epithelial cells using both in vitro and in vivo models. To our knowledge, this is the first work where these biological objects were analyzed both in vitro and in vivo with the same VLP batches.

\section{Material and Methods}

All reagents were obtained from Sigma-Aldrich (St. Louis, $\mathrm{MO})$, unless otherwise stated.

2.1. Antibodies. We used here previously tested antibodies against rotavirus or its structural proteins: anti-VP4 (clone 7.7) $[9,10]$, anti-VP2 (clone E22) [11], anti-VP6 (clone RV138) [12], anti-VP7 (M60) [13], anti RF (clone K2) [14, 15] Rabbit polyclonal anti-RF antiserum (8148) [9].

\subsection{Production and Purification of Complete and Incomplete} $V L P$. Nonreplicative rotavirus-derived VLP composed of VP2, VP4, and VP7 from bovine rotavirus RF strain and VP6 of simian rotavirus SA11 strain were produced and purified in our own laboratory using the baculovirus expression system as described elsewhere [4]. Briefly, Sf9 insect cells were coinfected with two recombinant baculoviruses expressing the GFP-VP2 fusion protein and the VP6 protein to generate incomplete VLP (VLP-GFP 2/6) or four recombinant baculoviruses expressing GFP: VP2, VP6, VP4, and VP7 viral proteins to generate complete VLP (VLP-GFP 2/6/7/4). Viral auto-assembled proteins were collected 6 days after infection from the $\mathrm{Sf} 9$ culture medium and purified by density gradient centrifugation in $\mathrm{CsCl}$. Finally the particles were resuspended in TNC buffer ( $50 \mathrm{mM}$ Tris, $150 \mathrm{mM} \mathrm{NaCl}$, $10 \mathrm{mM} \mathrm{CaCl}_{2}$ (pH 7.5)). The characterization of VLP was performed by Western Blot analysis and the concentration of proteins was determined using Quantity One analysis software (Bio-Rad Laboratories, France) and bovine serum albumin (BSA) (Pierce, Rockford, IL) as standard. Based on the number of molecules that compose the VLP and the molecular weight of each protein, it was assumed that 1$\mathrm{mg}$ of VLP suspension contains $\sim 1.2 \times 10^{13}$ particles $/ \mathrm{ml}$ [4]. GFP protein expression was quantified in 96 -well plates by spectrofluorometry using TECAN SpectraFluor (excitation filter: $485 \mathrm{~nm}$ and emission filter: $535 \mathrm{~nm}$ ) and the light emitted was recorded in arbitrary units using Xfluor Software V 3.21 (Tecan U.S., Research Triangle Park, NC). Appropriate assembly of VLP was confirmed by stoichiometry analysis and electron microscopy as previously described [4].

2.3. VLP Uptake by MA104 Cells. African green monkey kidney cells (MA 104) were grown as previously described [4]. For in vitro visualization, fluorescent (complete and incomplete) VLP previously treated with trypsin were incubated with MA104 epithelial cells for 1 hour at $4{ }^{\circ} \mathrm{C}$ and, since trypsin has been previously shown to be required to promote rotavirus cell entry $[16,17]$, nontrypsin treated VLP were used as control. VLP nonspecifically attached to the plasma membrane were removed by several washes, then incubation continued for 30 minutes at $37^{\circ} \mathrm{C}$. After incubation, cells were washed and fixed with $2 \%$ of paraformaldehyde (PFA) for 20 minutes at room temperature in the absence of any detergent to prevent cell permeabilization. Cells were washed again and incubated with a red fluorescent lectin, Wheat Germ Agglutinin (WGA) conjugated with Alexa Fluor 594 (Molecular Probes, Invitrogen France) in order to visualize the cell surface. Labeled cells were observed by confocal microscopy. The total fluorescence was quantified using the built-in Leica software that allows to measure specific-over-background fluorescence on a defined region of interest (ROI). Specifically, the ROI was defined as the entire microscopic field in each of the five independent experiments and we have compared the total green fluorescence at the different time.

2.4. In Vivo VLP Uptake by Mice Intestinal Cells. Seven week-old male Balb/c mice were purchased from Centre d'Elevage René Janvier, (Le Genest Saint-Isle, France) and were housed in groups of four mice per cage in pathogenfree Texler-type isolators (La Calhene, Velizy, France) under sterile conditions with water and were fed ad libitum in the animal facilities of the Unité d'Ecologie et de Physiologie du Système Digestif at the Institut National de la Recherche Agronomique (INRA, Jouy-en-Josas, France). Experiments were started after one week of acclimatization and performed according to international rules of animal care. To respect the safe volume to gavage in mice, groups of mice were inoculated by intragastric gavage with two consecutive doses of complete or incomplete VLP $\left(1 \times 10^{12}\right.$ particles $\left./ \mathrm{ml}\right)$ at 2hour intervals in order to allow mice to recover. TNC buffer was used as a negative control. Four hours after the last administration, mice were sacrificed by vertebral dislocation; the intestine was removed under sterile conditions and cut into regional sections: ileum, proximal colon and distal colon. Each section was flushed out with phosphate buffer solution (PBS, Gibco BRL, France) as described elsewhere [18]. For the ELISA assay, $\sim 2 \mathrm{~cm} \times 1 \mathrm{~cm}$ pieces of tissue sample (ileum, proximal or distal colon) were cut, washed vigorously and placed into $1.5 \mathrm{ml}$ of PBS containing antiproteases (Protease Inhibitor Cocktail, Complete mini tablets, Roche Diagnostics, France). The samples were homogenized using a Polytron homogenizer and cell debris eliminated by centrifugation; supernatants were recovered and stored at $-80^{\circ} \mathrm{C}$. For microscopic examination, ileum and colon samples were recovered cut longitudinally, cleaned and 


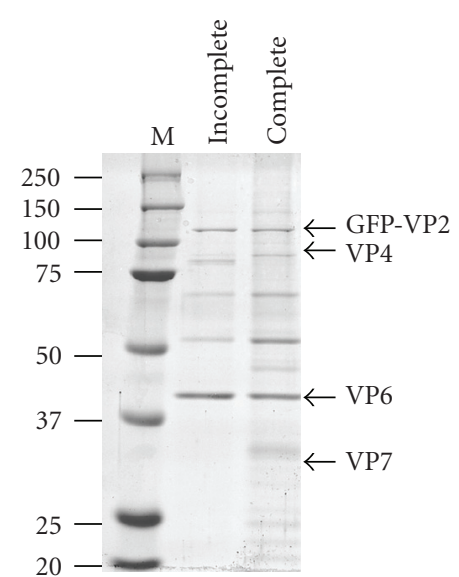

(a)

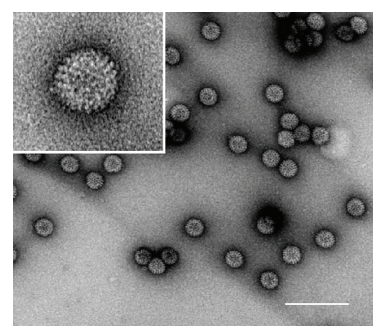

(c)
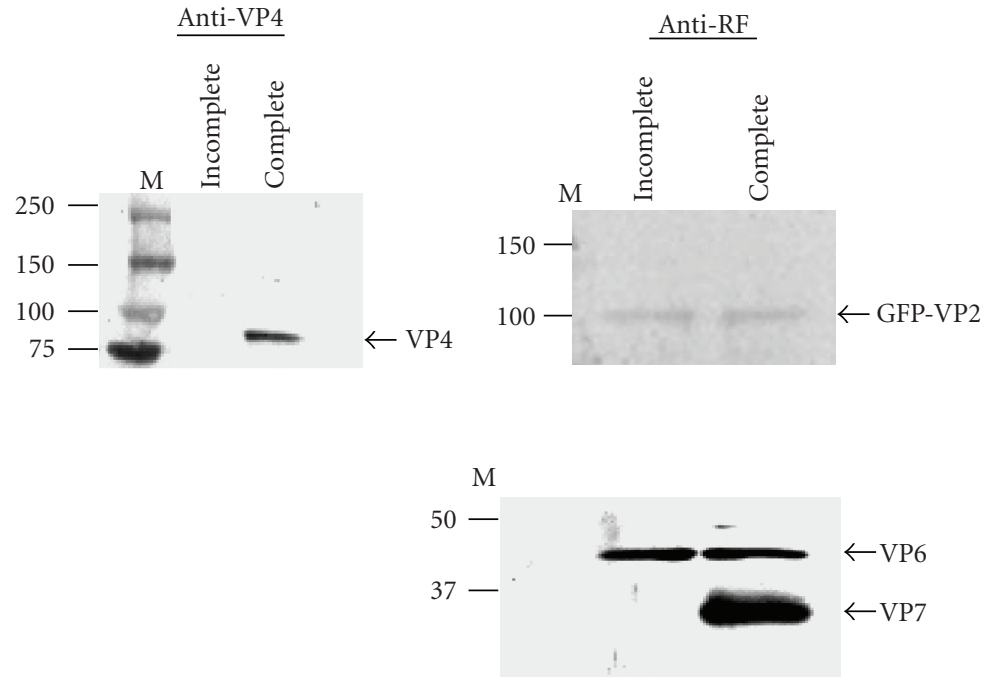

(b)

Figure 1: Analysis of VLP purified from baculoviruses infected Sf9 insect cells. Incomplete and complete VLP from the culture medium of Sf9 cells were purified using $\mathrm{CsCl}$ gradients. (a) Coomassie blue-stained SDS-PAGE showing incomplete and complete VLP containing GFP : VP2 fusion proteins. (b) Western-blot of recombinant VLP proteins using either anti-VP4 or anti-RF rotavirus antibodies. M: protein molecular weight markers. (c) Electron microscopy analysis of incomplete VLP negatively stained with a 2\% uranyl acetate solution, scale bar $100 \mathrm{~nm}$. Insert: higher magnification of a single VLP.

frozen in liquid nitrogen. Intestinal samples were then sliced into $5 \mu \mathrm{m}$ sections and stored at $-20^{\circ} \mathrm{C}$ until analysis.

2.5. Mouse Model of Inflammation. Twelve mice (colitis group) were anaesthetized with isoflurane and received intrarectally $100 \mu \mathrm{l}(100 \mathrm{mg} / \mathrm{kg})$ of 2,4,6-trinitrobenzene sulfonic acid (TNBS, Fluka, France) in 50\% ethanol as previously described [19]. Twelve other mice (colitis free group) served as naïve control. Two days after TNBS administration in the colitis group, mice of each group were randomized into three different subgroups to receive complete VLP, incomplete VLP, or TNC buffer as above described. After sacrifice, a macroscopic evaluation of colitis damage was performed according to the Wallace criteria [20].

2.6. ELISA. For ELISA measurements, the concentrations of total proteins in homogenate samples from intestine were determined using the Bicinchoninic acid (BCA) protein assay reagent (Pierce, Rockford, IL) and protein concentrations adjusted at $100 \mu \mathrm{g} / \mathrm{ml}$ and $400 \mu \mathrm{g} / \mathrm{ml}$, respectively. Ninetysix well plates Nunc Maxisorp (Nunc, Roskilde, Denmark) were coated with antirotavirus antibodies (see above) diluted
$1: 1000$ in sodium carbonate buffer $(50 \mathrm{mM}$ sodium carbonate, $1 \mathrm{mM} \mathrm{MgCl}_{2} \mathrm{pH} 9.8$ ) and incubated overnight at $4^{\circ} \mathrm{C}$. Plates were washed twice with PBS containing $0.05 \%$ Tween-20 (PBS-T). Wells were then blocked with blocking buffer (PBS-T containing 3\% bovine serum albumin) for 2 hours at room temperature and washed three times with PBS-T. A $100 \mu \mathrm{l}$ sample previously diluted in blocking buffer was added to each well and incubated 2 hours at room temperature. After three washing steps, anti-RF rabbit polyclonal antibody (kindly provided by Dr R. L'Haridon, INRA Jouy en Josas, France) diluted 1:500 in blocking buffer was added and the plate incubated for 1 hour at room temperature. The plates were washed as described above and horseradish peroxidase conjugated (HRP) goat anti-rabbit IgG antibodies (Rockland, Gilbertsville, PA) diluted 1:5000 in blocking buffer was added to each well and incubated for 1 hour at room temperature. After 3 washes, the reaction was developed by addition of 3,3',5,5' -tetramethylbenzidine substrate (TBM, Sigma-Aldrich, St. Louis, MO). Finally the reaction was stopped by the addition of $\mathrm{H}_{2} \mathrm{SO}_{4}$ and the absorbance was immediately measured at $450 \mathrm{~nm}$.

The amount of green fluorescent protein (GFP) was assessed by ELISA using React-bind anti-GFP coated plates 


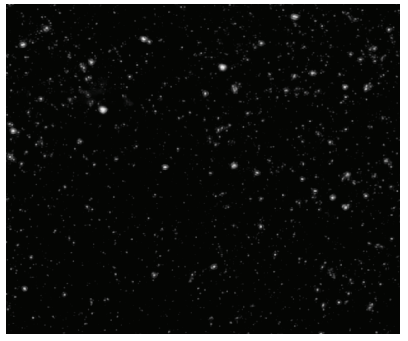

GFP-VP2 VLPs

(a)

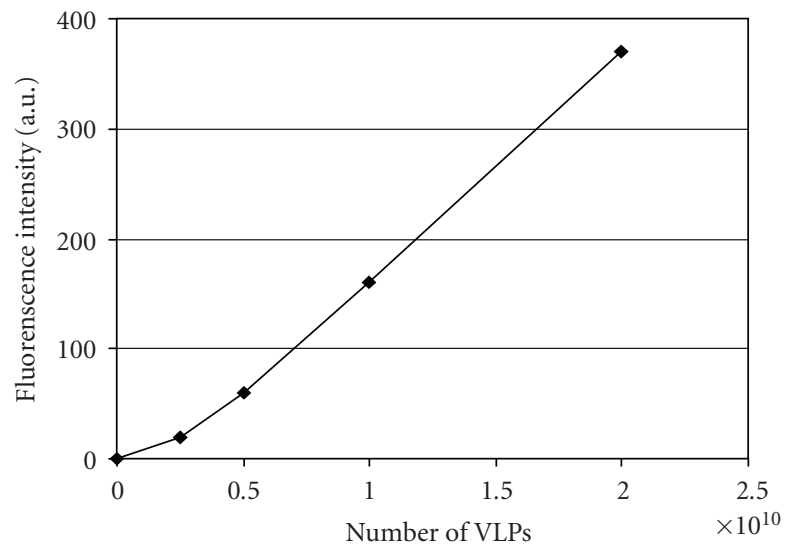

(b)

FIGURE 2: Quantification of VLP carrying GFP: VP2 fusion protein. (a) $\mathrm{CsCl}$ purified fluorescent VLP were concentrated by centrifugation and the pelleted VLP recovered in TNC buffer, spotted on glass slides, and visualized by confocal microscopy. TNC buffer was used as a control. (b) Increasing amounts of VLP-GFP, as measured by protein concentration, were placed in 96-well plaques and fluorescence intensity was measured by direct spectrofluorometry using a TECAN SpectraFluor. Results are representative of 3 independent experiments.

(Pierce, Rockford, IL). Briefly, a $100 \mu \mathrm{l}$ sample was coated for 1 hour at room temperature. Plates were washed; rabbit antiGFP serum (kindly provided by Dr J.-L. Delaunay, INSERMUPMC UMRS538, Paris, France) diluted $1: 1000$ in blocking buffer was added and incubated for 1 hour. HRP conjugated goat anti-rabbit, and the TBM substrate was then added as above described.

2.7. Confocal Microscopy. Fixed MA104 cells were examined for WGA and VLP-GFP using a Leica TCS Spectral (SP2) instrument equipped with an inverted microscope using $63 \times$ and $100 \times$ oil immersion objectives. Fluorescence intensity of VLP-GFP was quantified using on-line "Scan Ware" software. Cryosections from mice intestines were fixed in acetone for 10 minutes, air-dried, rinsed twice with PBS, treated with retrieval buffer (PBS pH 7.4 containing $0.3 \%$ Triton $\mathrm{X}-100$ and $1 \%$ bovine serum albumin) and blocked with blocking solution (PBS with $1 \%$ bovine serum albumin). The primary rabbit anti-RF antibody was used at $1: 500$. Indirect fluorescence was carried out using Cy3conjugated goat anti-rabbit (Jackson Immunoresearch, West
Grove, PA). Double-fluorescence acquisition was performed for colocalization studies of viral proteins and GFP using the confocal microscope. In some experiments, FITC Conjugated Datura stramonium (Lectin DSA, EY Laboratories Inc.) was used as a green-fluorescence positive control to visualize the gut.

2.8. Statistical Analyses. Data were analyzed using StatDirect Statistical software, version 2.6.5 (http://www.statsdirect .com). 95\% confidence limits, Tukey-Kramer test was used to compare the difference between means. In some experiments, ANOVA followed by Dunnett's test was used to compare different treatments with the control group. A value of $P<.05$ was considered as significant.

\section{Results}

3.1. Production and Characterization of Fluorescent VLP. In order to determine whether Sf9 cells coinfected with baculoviruses expressing rotavirus proteins were able to generate incomplete and complete VLP, samples were collected and purified after infection and tested for viral proteins by SDSPAGE (Figure 1(a)) and Western Blot analysis (Figure 1(b)). Anti-VP4 and anti-RF antibodies revealed an appropriate production of proteins in both incomplete (GFP-VLP 2/6) and complete (GFP-VLP 2/6/7/4) VLP. In addition, analysis by electron microscopy was performed to confirm the correct assembly and structure of VLP (Figure 1(c)). GFP concentration was calculated using the same method described above for VLP. It is assumed that a VLP contain 120 VP2 molecules, thus 120 molecules GFP [4]. Consequently, we estimated the concentration of GFP at $\sim 8 \mu \mathrm{g} / \mathrm{VLP}$. The correct conformation of GFP from GFP : VP2 fusion protein within VLP was confirmed by direct analysis under fluorescence microscopy (Figure 2(a)). Spectrofluorometry was used to show that the number of VLP particles; GFP fluorescence was a linear function of VLP concentration (Figure 2(b)). The relative fluorescence is in agreement with the generation of GFPassociated VLP.

3.2. Entry of VLP into Cultured Epithelial Cells. To explore the capacity of VLP to deliver exogenous proteins to epithelial cells, interaction experiments were first performed between trypsin-treated VLP and cultured MA104 epithelial cells. The results obtained revealed that after a 20-minute exposure period of trypsin-treated VLP with MA104 cells, only complete VLP efficiently enter into cells, whereas incomplete (data not shown) as well as trypsin untreated VLP do not significantly penetrate (Figure 3(a)). Internalization of VLP was first evaluated as the percentage of reduction of GFPVLP at the cell surface as measured by fluorescence intensity quantification. After a 20 -minute exposure period, only $50 \%$ of the initial fluorescence were recovered when trypsintreated VLP were used, whereas there was no significant decrease with untreated VLP (Figure 3(b)). In a second experiment, we measured the amount of GFP that accumulated within MA104 cell homogenates by using ELISA. As shown in Figure 3(c), GFP was easily detectable after the 


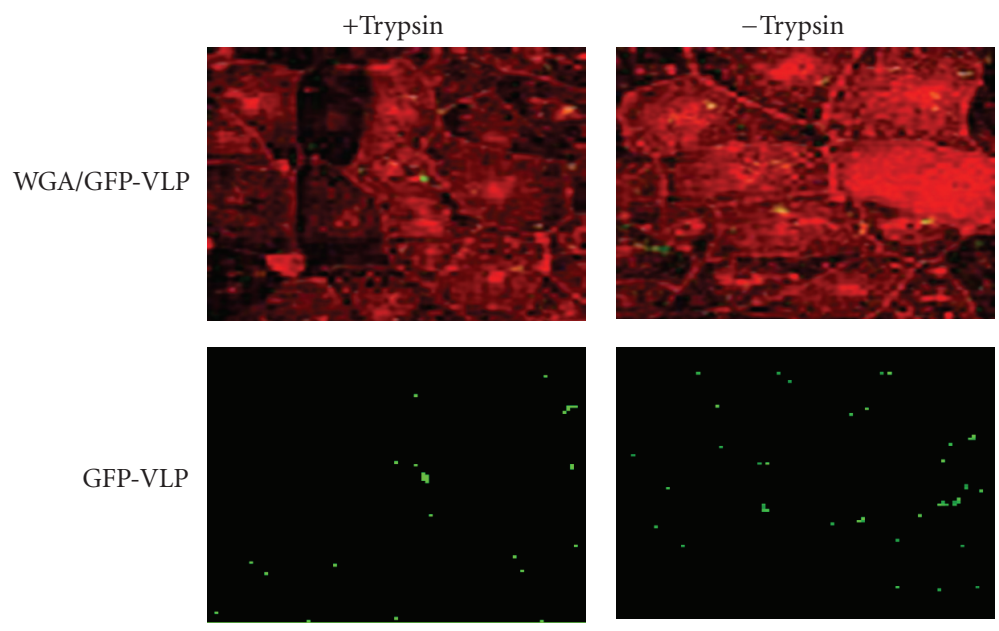

(a)

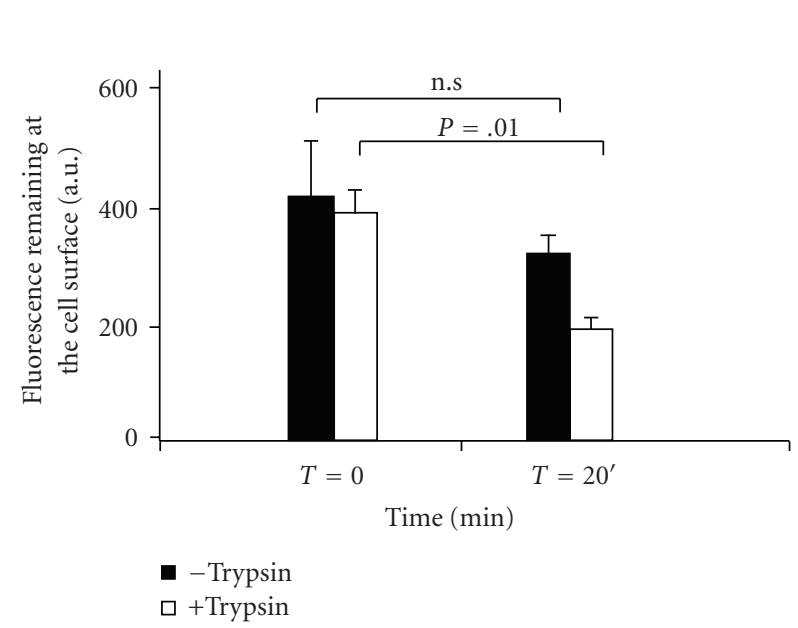

(b)

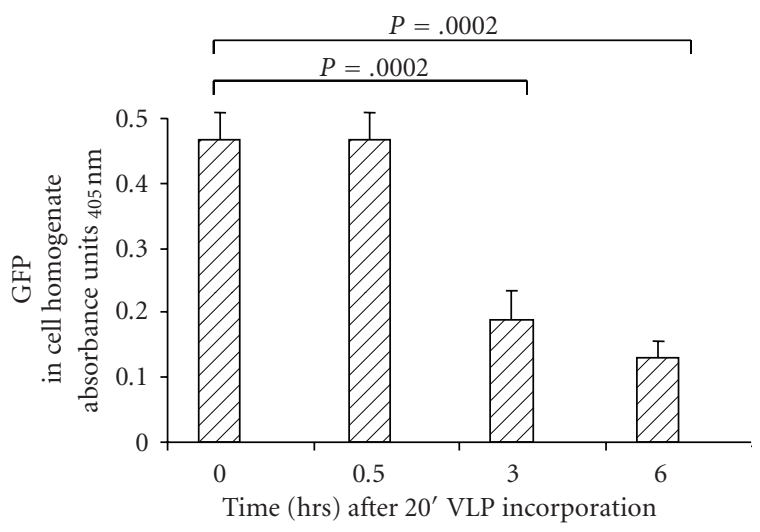

(c)

FIGURE 3: Efficiency of VLP internalization in intestinal epithelial cells in vitro. (a) Fluorescence microscopy of MA104 cells after 20-minute exposure to complete GFP-VLP (green label) treated or not with trypsin. MA104 cell surface was labeled using WGA lectin (red label). A representative image of 5 independent experiments is shown. (b) Internalization was quantified by measuring the GFP fluorescence at the cell surface using the built-in Leica software. Results are expressed as the amount of GFP fluorescence (a.u.) at the cell surface as a function of time. Results are means of 5 independent experiments. (c) Detection of GFP within cell homogenates using ELISA assay. GFP amounts were determined immediately after the initial 20-minute interaction between cell and GFP-VLP (time 0 ) and then for the indicated times. Samples were tested in triplicate in two independent experiments. Results are expressed as the mean absorbance value \pm standard deviation (sd).

initial 20-minute exposure period of VLP to MA104 cells. It is important to note here that the amount of immunoreactive GFP within cell homogenates showed a $\sim 75 \%$ drop of GFP, six hours after contact with trypsin-treated VLP. This result suggests that the GFP should probably be processed once inside the cell.

\subsection{Delivery of GFP by VLP into Intestinal Cells In Vivo.} Once it was established that complete VLP could deliver GFP into MA104 cells, we next explored the capacity of these particles to deliver GFP into intestinal cells in vivo. Confocal analysis of intestinal samples from mice administered with complete VLP resulted, as expected, in the detection of
GFP (green) that colocalized (yellow) with viral proteins labeled with anti-RF rotavirus antibodies (red) in the ileum (Figure 4(a)). Unexpectedly, we also observed the presence of VLP within colonic epithelial cells in these in vivo experiments (Figure $4(\mathrm{~b})$ ). To confirm our observations, viral proteins were quantified in mice ileum and colon homogenates using ELISA. As expected from the above experiments, we observed the presence of viral proteins VP4, VP2, and VP6 in the ileum suggesting a significant entry of complete VLP, whereas incomplete VLP were unable to significantly penetrate the ileum (Figure 5). Using antiVP7 determination, we estimate that $\sim 42 \%\left(2.9 \times 10^{9}\right.$ VLP $)$ entered in ileum and $\sim 30 \%\left(2.1 \times 10^{9} \mathrm{VLP}\right)$ in colon. More 

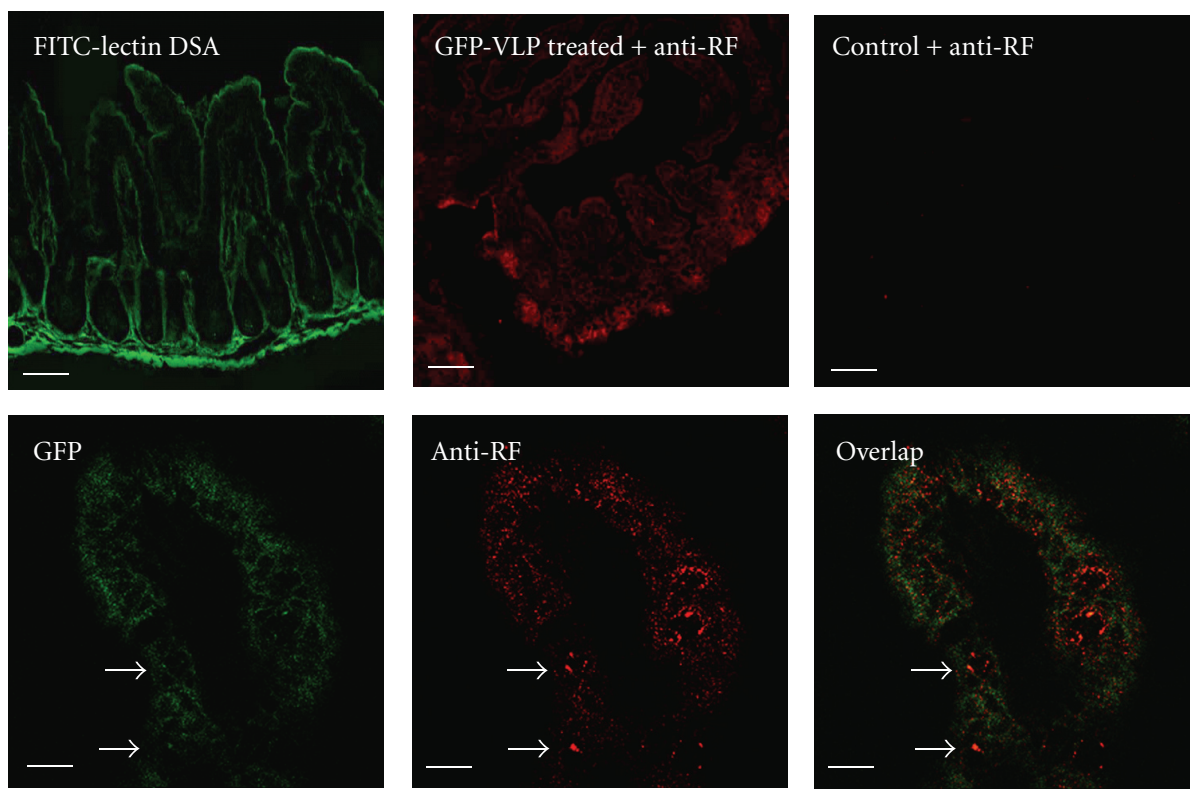

(a)
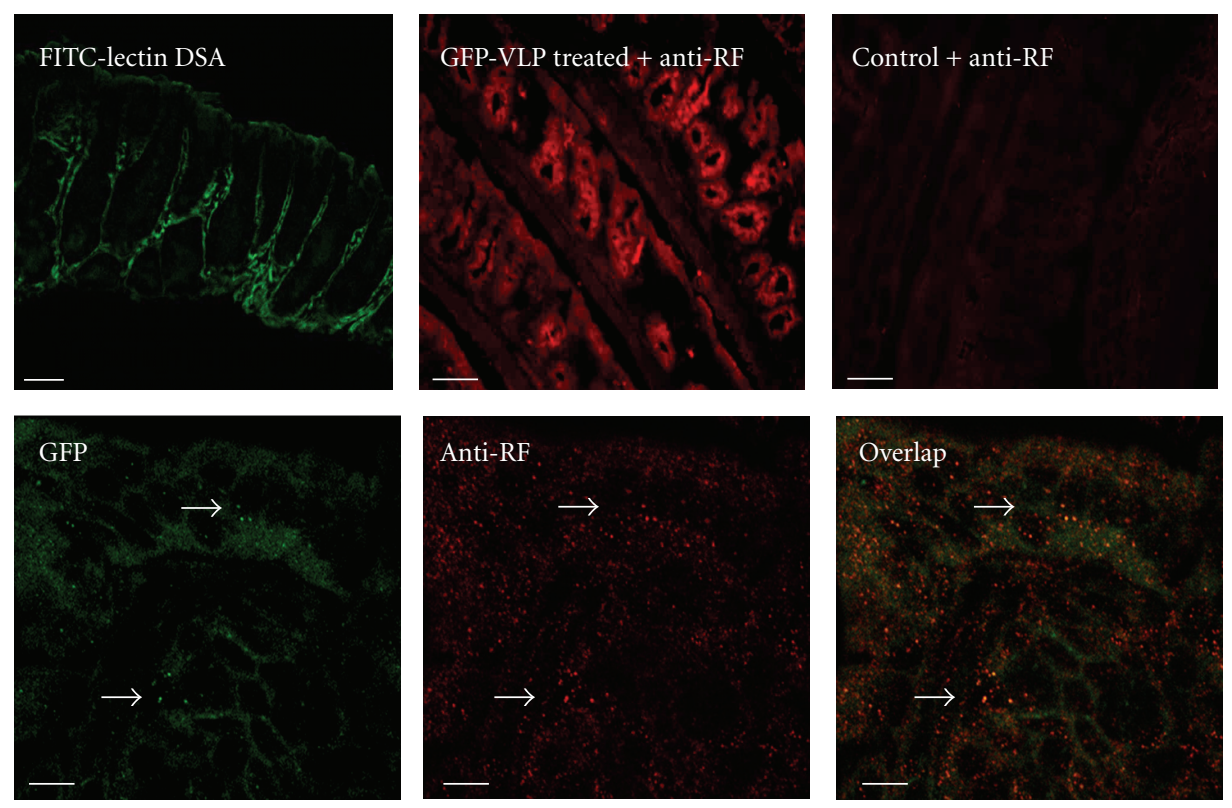

(b)

FIGURE 4: Complete VLP internalize in vivo in the ileum (a) and colon (b). Frozen sections of intestinal samples from mice administered with complete GFP-VLP (green) were labeled with anti-RF antibodies and Cy3-conjugated (red); FITC-lectin DSA was used as a positive control. Tissue sections were analyzed by confocal microscopy. Overlay analysis shows that GFP colocalizes with viral proteins (yellow/orange). The arrows indicate the VLP. Representative images of 3 experiments are shown. Scale bar $20 \mu \mathrm{m}$.

surprisingly a modest amount of VLP was recovered within the colon and a very significant amount of VP2 was detected within the colon when incomplete VLP was used. It is worth noting that using the protocol described above, the limit of detection using these antibodies is of $\sim 9.3 \times 10^{9}$ to VP4, VP2 and VP6 and $\sim 1 \times 10^{9}$ to VP7.

3.4. Delivery of GFP by VLP into Intestinal Cells In Vivo under Inflammatory Conditions. To determine whether our approach could be used to deliver bio-active proteins into intestinal cells under in vivo inflammatory conditions, we assessed GFP delivery by VLP in a mouse model of intestinal inflammation (TNBS model, see material and methods). Groups of mice were slightly anesthetized and treated with TNBS. Two days after, when the inflammation was established, complete and incomplete VLP were administered, tissue samples were collected and analyzed as indicated in Figure 6(a). As expected, TNBS-treated mice presented the common symptoms of colitis: hyperemia, ulcerations, intestinal damage and assessed using the Wallace Score 

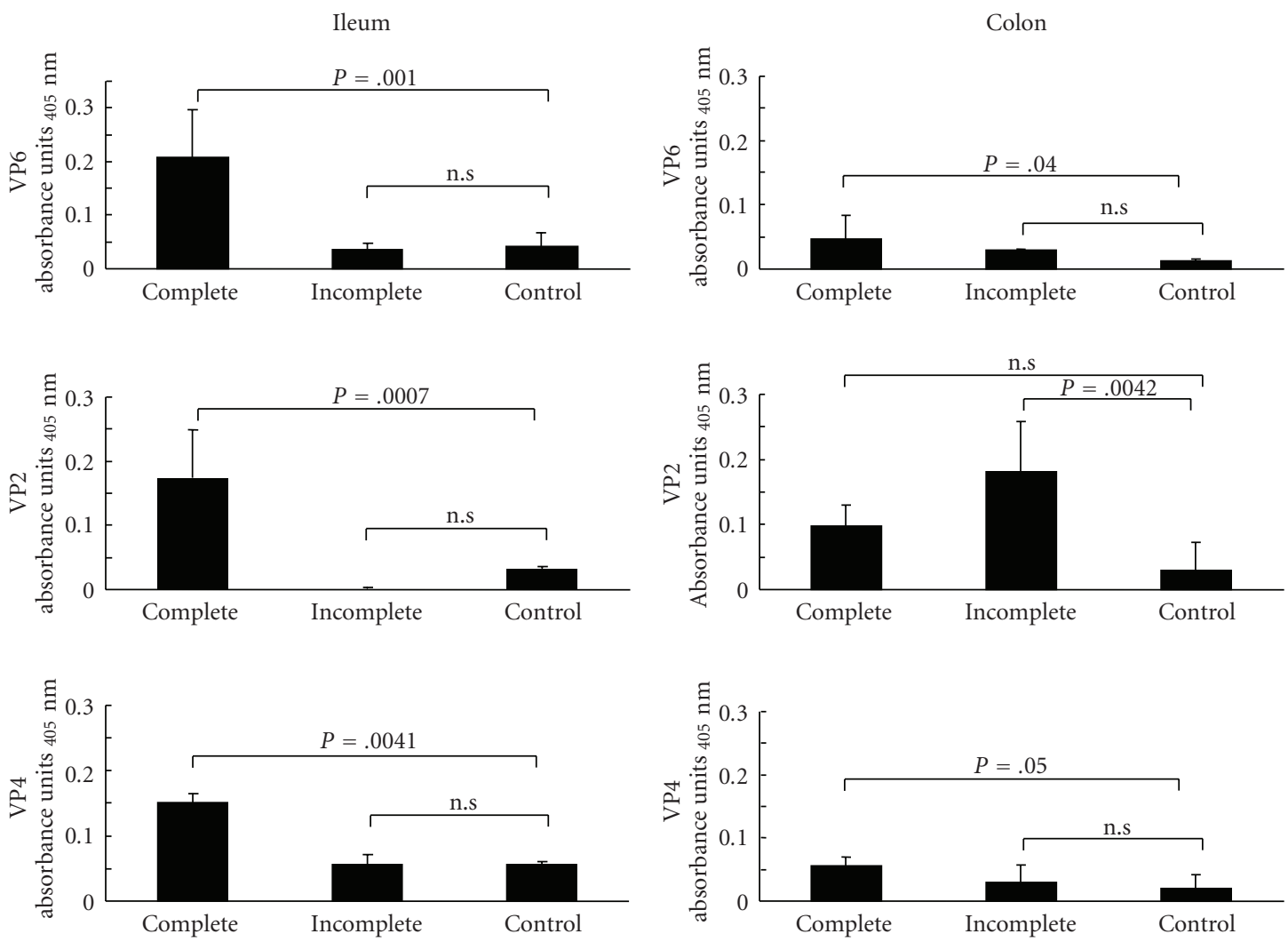

FIGURE 5: Quantification of viral proteins internalized in intestinal sections after VLP administration to living mice. The presence of VP2, VP4, and VP6 rotavirus proteins in the ileum and the colon of mice administered with complete VLP $(n=4)$ was evaluated by ELISA. Results are expressed as absorbance units and are the mean $\pm \mathrm{sd}$. Dunnett's test was used to compare treatments with the control group.

(Figure 6(b)) and a diminution in colon length (Figure 6(c)). None of these inflammation markers was influenced by the presence of complete or incomplete VLP (Figure 6(b)). Once inflammation was confirmed in TNBS treated mice, we evaluated the ability of complete and incomplete VLP to transfer the reporter protein by measuring GFP amounts in gut homogenates using ELISA. The results revealed the presence of significant amounts of GFP and viral proteins in colon samples of both TNBS-treated and normal mice (Figures $7(\mathrm{a})$ and $7(\mathrm{~b})$ ). In contrast, whereas viral proteins were detected (Figure 7(c)), we were unable to detect significant amounts of GFP within the ileum. The current study indicated that GFP-VLP entered both the ileum and the colon and that the reporter protein was detectable in normal and inflamed colon but not in the ileum.

\section{Discussion}

In this work, we show that nonreplicative trypsin-treated VLP do penetrate into cultured cells and, when given intragastrically to living mice, can also enter into both normal and inflamed intestinal epithelium. Our results also show that VLP can transport biomolecules such as GFP to specific sites both in vivo and in vitro. This represents a potential original new nanoparticle system for delivering bioactive molecules directly into intestinal cells.

Our in vitro experiments using epithelial MA104 cells show an efficient internalization $(\sim 50 \%)$ of trypsin-activated complete VLP after only 20 minutes of interaction. These observations are consistent with previously reported findings and confirm that the cleavage of the rotavirus spike protein VP4 is a prerequisite for cellular internalization $[4,16]$. According with previously reported findings, we observe that absence of VP4 and VP7 does not modify cell attachment [4]. However, several investigations have already demonstrated the key role of the tryptic cleavage of VP4 for cellular entry $[16,17,21-24]$. Thus, according to these observations, our results confirm that nonreplicative VLP display attachment and entry properties very similar to those of authentic rotavirus.

After administration of complete GFP-VLP to mice, and as expected given the homology with infectious rotavirus, we observed the delivery of complete but not of incomplete VLP to the ileum, the natural target of this virus by fluorescence microscopy and ELISA detection of viral proteins within cell homogenates. In addition, it is worth noting that efficacy of bovine Rotavirus heterologous infection has not been well studied. Recently, Istrate $\mathrm{C}$ and collaborators report for the first time that a bovine Rotavirus strain is capable of infecting 


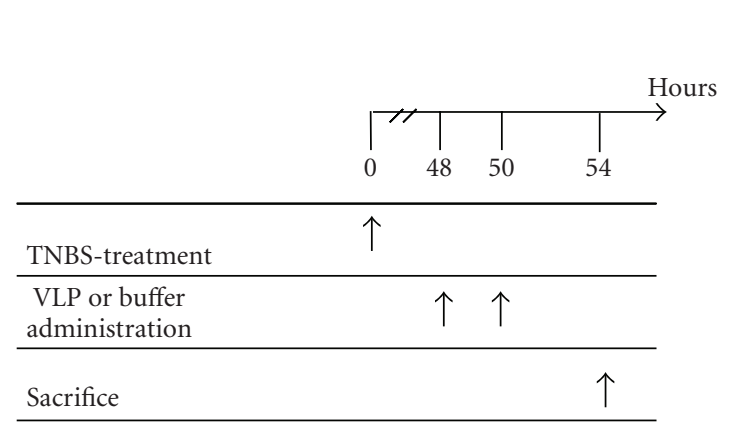

(a)

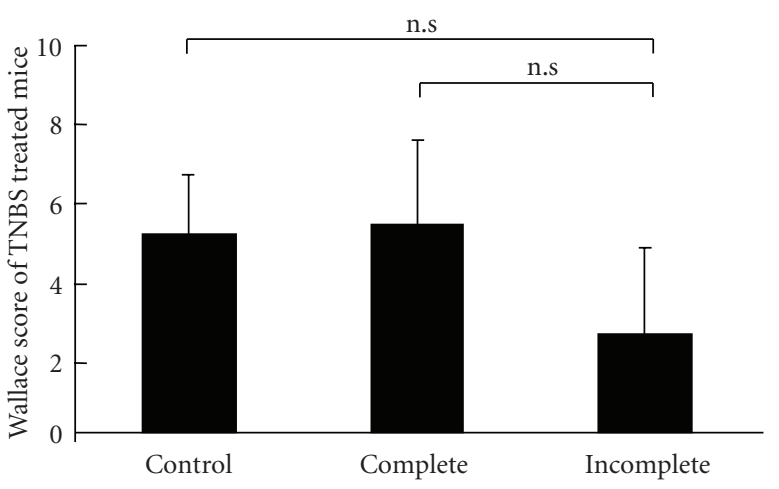

(b)

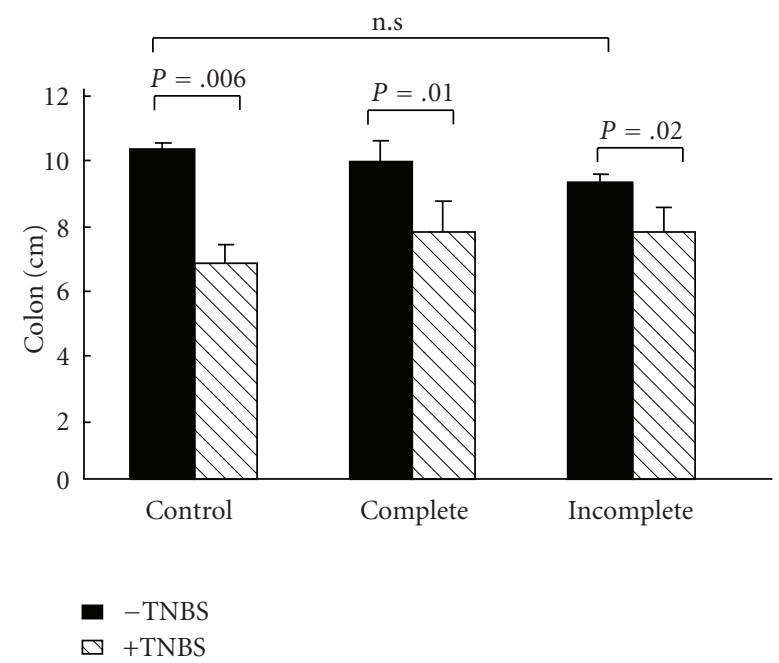

(c)

FIGURE 6: TNBS-induced colitis in the mouse model. (a) Scheme of the experimental protocol used for the induction of colitis in mice and time of complete or incomplete VLP administration, buffer administration, and sacrifice. (b) Colonic macroscopic damage according to the criteria of Wallace of TNBS-treated animals $(n=4)$. (c) Comparison of colon length between control and TNBS-treated mice $(n=4)$. Tukey-Kramer test was used to compare the difference between means. Results are expressed as the means \pm sd.

murine bmDC in vitro [25]. Likewise Guerin-Danan et al. reported the capacity of simian SA11 to infect rats [26]. Our results strongly suggest that VLP enter ileum through the classical route that strictly depends on the presence of cleaved VP4 and VP7 capsid proteins. Thus provide evidence of "in vivo" heterologous Rotavirus pseudoinfection. However, despite this obvious entry process of complete VLP, we were unable to detect GFP within the ileum by ELISA. During the in vivo experiments, the time between VLP administration and sample processing was at least 4 hours, that is, the time after which the large majority of GFP has disappeared in the in vitro experiments. The absence of detection of GFP within the ileum is likely due to the fact that the protein was already delivered to an intracellular compartment where it suffers an alteration either in the conformation or in the concentration and not only due to a subcellular localization where fluorescence can be quenched, as proposed earlier [4]. In the meantime, it should be emphasized that viral structural proteins that have also entered within mice ileum are still significantly expressed in the tissue. This means that the fate of each particular protein that has entered the ileum should be specific. Further development of our work have to analyze this point carefully in order to add targeting information to a putative vectorized therapeutic protein allowing to escape this denaturation/degradation event.

Astonishingly, the results indicate that, in the colon, a significant entry of complete VLP happens and even more that an uptake of incomplete VLP may also be observed, as assessed by the detection of the internal capsid protein VP2 within colon tissue homogenate. In addition, GFP was efficiently delivered to colonic tissues either with complete or incomplete VLP-GFP. Entry of a small amount of complete VLP within the colon results in a very efficient delivery of the reporter protein. Comparison of these data with those observed in the ileum leads to the suggestion that complete VLP enter the colon through an alternative route, driving the VLP to an intracellular compartment where no subsequent GFP modifications can occur. Such a hypothesis is highly plausible given the cell populations present in the colon as compared to the ileum. In this regard, it has 


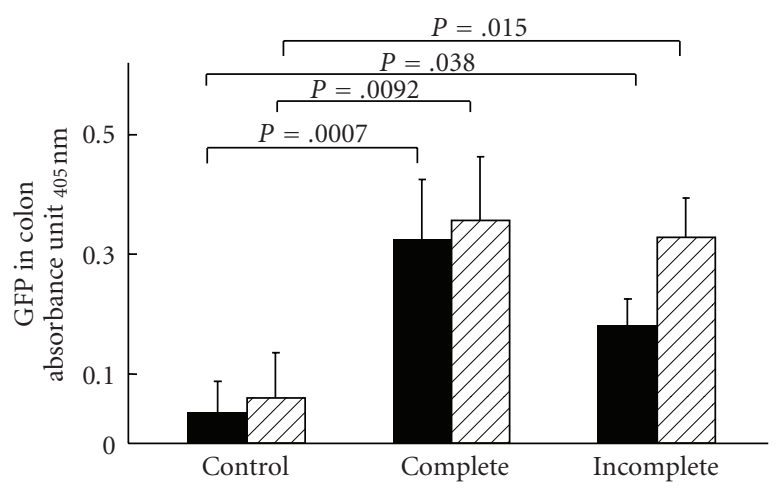

(a)

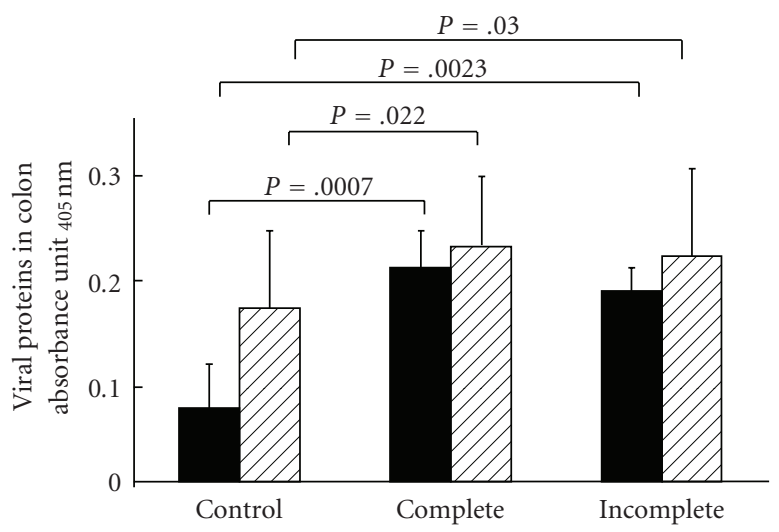

(b)

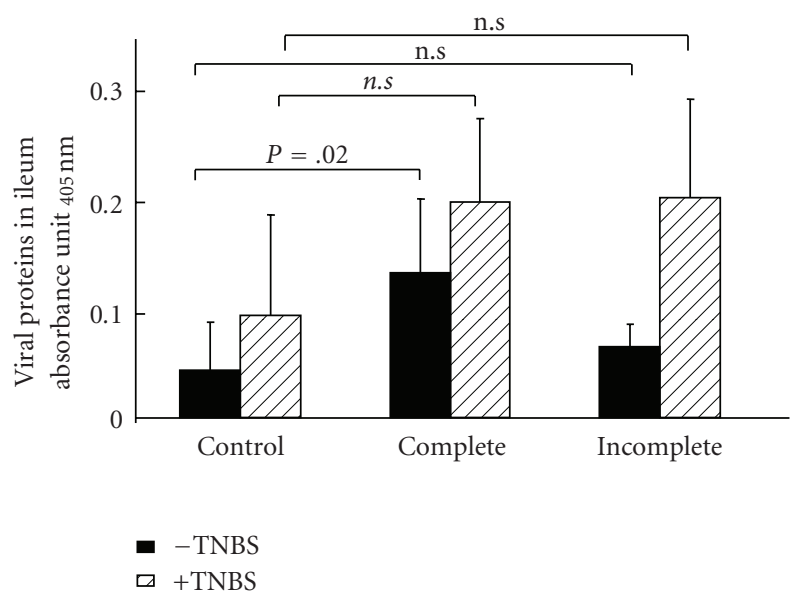

(c)

FIGURE 7: Complete or incomplete VLP delivery to the normal or inflamed gut. Delivery of GFP (a) and viral proteins (b) to the colon and of viral proteins to the ileum (c) from healthy and TNBS-treated mice $(n=4)$ was evaluated by ELISA assays. Results are expressed as absorbance units and are the mean $\pm \mathrm{sd}$ of three experiments. Dunnett's test was used to compare treatments with the control group.

already been shown that complete VLP, but not incomplete VLP were able to enter in dendritic cells "in vitro" [4]. In addition, previously reports have shown that rotavirus preferentially targeted to absorbing intestinal cells rather than Peyer's Patches in suckling rats [26]. Although the precise identification of this compartment and/or the cell types implied remains to be determined, our results show that only complete VLP are able to enter into the ileum. This work represents the first study of VLP interaction "in vivo" and clearly demonstrates that complete VLP represent a new efficient tool to deliver a biomolecules to the colon.

In the case of incomplete VLP, the mechanism of GFP transfer into the colon appears different, since our results indicate that only VP2 (and GFP) can be detected in these conditions within the colonic tissue. A possible explanation for these results may be that VP6 into incomplete VLP are much more accessible to intraluminal degradation than complete VLP; consequently, VP2: GFP enters alone within colonic cells. If this is the case, VP2 may represent an interesting vector protein.

Finally, one of our goals was to demonstrate that VLP may be used in both healthy and inflamed intestinal tissues.
Thanks to the TNBS model, we were able to generate a true colitis and to check the capacity of VLP to transfer GFP into both normal and inflamed mice colons. Our results clearly indicate that GFP was efficiently transferred within the colon in the two situations, thus opening the possibility to further elaborate this new and original delivery vector with the aim to propose innovative treatments for IBD.

\section{Acknowledgments}

The authors would like to thank Annie Charpilienne (INRA, Gif sur Yvette, France) for providing us with a set of recombinant baculovirus and related antibodies, René L'Haridon (INRA, Jouy en Josas, France) for providing antibodies, as well as Sebastien Blugeon (INRA, Jouy en Josas, France), Violaine Rochet (INRA, Jouy en Josas, France), and Christine Longin (INRA MET-Jouy en Josas) for technical assistance. This work was in part supported by grants of UCB Pharma, The Association François Aupetit (AFA), ACI Nanosciences and Institutional funding by INSERM and UPMC. This work is dedicated to the memory of the author's friend Jean Cohen. 


\section{References}

[1] J. A. Wolff, "The "grand" problem of synthetic delivery," Nature Biotechnology, vol. 20, no. 8, pp. 768-769, 2002.

[2] M. T. Lotze and T. A. Kost, "Viruses as gene delivery vectors: application to gene function, target validation, and assay development," Cancer Gene Therapy, vol. 9, no. 8, pp. 692-699, 2002.

[3] C. Van Montfrans, A. A. te Velde, S. J. H. van Deventer, and M. S. Rodriguez Pena, "Gene therapy in the treatment of intestinal inflammation," International Journal of Colorectal Disease, vol. 19, no. 2, pp. 79-86, 2004.

[4] A. Charpilienne, M. Nejmeddine, M. Berois, et al., "Individual rotavirus-like particles containing 120 molecules of fluorescent protein are visible in living cells," The Journal of Biological Chemistry, vol. 276, no. 31, pp. 29361-29367, 2001.

[5] C. Q.-Y. Zeng, M. J. Wentz, J. Cohen, M. K. Estes, and R. F. Ramig, "Characterization replicase activity of doublelayered and single-layered rotavirus-like particles expressed from baculovirus recombinants," Journal of Virology, vol. 70, no. 5, pp. 2736-2742, 1996.

[6] C. Q.-Y. Zeng, M. K. Estes, A. Charpilienne, and J. Cohen, "The N terminus of rotavirus VP2 is necessary for encapsidation of VP1 and VP3," Journal of Virology, vol. 72, no. 1, pp. 201-208, 1998.

[7] M. K. Estes, J. M. Ball, S. E. Crawford, et al., "Virus-like particle vaccines for mucosal immunization," Advances in Experimental Medicine and Biology, vol. 412, pp. 387-395, 1997.

[8] J. M. Gilbert and H. B. Greenberg, "Virus-like particleinduced fusion from without in tissue culture cells: role of outer-layer proteins VP4 and VP7," Journal of Virology, vol. 71, no. 6 , pp. 4555-4563, 1997.

[9] M. Nejmeddine, G. Trugnan, C. Sapin, et al., "Rotavirus spike protein VP4 is present at the plasma membrane and is associated with microtubules in infected cells," Journal of Virology, vol. 74, no. 7, pp. 3313-3320, 2000.

[10] O. Delmas, M. Breton, C. Sapin, A. Le Bivic, O. Colard, and G. Trugnan, "Heterogeneity of raft-type membrane microdomains associated with VP4, the rotavirus spike protein, in Caco-2 and MA 104 cells," Journal of Virology, vol. 81, no. 4, pp. 1610-1618, 2007.

[11] A. Roseto, R. Scherrer, and J. Cohen, "Isolation and characterization of anti-rotavirus immunoglobulins secreted by cloned hybridoma cell lines," Journal of General Virology, vol. 64, no. 1, pp. 237-240, 1983.

[12] P. Pothier, F. Limone, E. Kohli, S. Ghim, and J. B. Bour, "Development and preliminary evaluation of a latex agglutination test using a monoclonal antibody for rotavirus detection in stool specimens," Annales de l'Institut Pasteur Virology, vol. 138, no. 4, pp. 523-526, 1987.

[13] R. D. Shaw, P. T. Vo, P. A. Offit, B. S. Coulson, and H. B. Greenberg, "Antigenic mapping of the surface proteins of rhesus rotavirus," Virology, vol. 155, no. 2, pp. 434-451, 1986.

[14] B. Akindes, A. Roseto, C. Feynerol, and R. Scherrer, "Obtention et Caracterisation d'anticorps monoclonaux diriges contre les rotavirus humain et bovine," Colloque d l'Inserm sur la Diarrhee du Jeune, vol. 121, pp. 345-358, 1984.

[15] R. L'Haridon and R. Scherrer, "In vitro culture of a rotavirus associated with neonatal calf diarrhea," Annales de Recherches Veterinaires, vol. 7, no. 4, pp. 373-381, 1976.

[16] C. F. Arias, P. Romero, V. Alvarez, and S. López, "Trypsin activation pathway of rotavirus infectivity," Journal of Virology, vol. 70, no. 9, pp. 5832-5839, 1996.
[17] P. R. Dormitzer, E. B. Nason, B. V. V. Prasad, and S. C. Harrison, "Structural rearrangements in the membrane penetration protein of a non-enveloped virus," Nature, vol. 430, no. 7003, pp. 1053-1058, 2004.

[18] N. G. Cortes-Perez, F. Lefevre, G. Corthier, K. Adel-Patient, P. Langella, and L. G. Bermúdez-Humarán, "Influence of the route of immunization and the nature of the bacterial vector on immunogenicity of mucosal vaccines based on lactic acid bacteria," Vaccine, vol. 25, no. 36, pp. 6581-6588, 2007.

[19] B. Foligne, S. Nutten, L. Steidler, et al., "Recommendations for improved use of the murine TNBS-induced colitis model in evaluating anti-inflammatory properties of lactic acid bacteria: technical and microbiological aspects," Digestive Diseases and Sciences, vol. 51, no. 2, pp. 390-400, 2006.

[20] J. L. Wallace, W. K. MacNaughton, G. P. Morris, and P. L. Beck, "Inhibition of leukotriene synthesis markedly accelerates healing in a rat model of inflammatory bowel disease," Gastroenterology, vol. 96, no. 1, pp. 29-36, 1989.

[21] C. Sánchez-San Martín, T. López, C. F. Arias, and S. López, "Characterization of rotavirus cell entry," Journal of Virology, vol. 78 , no. 5, pp. 2310-2318, 2004.

[22] M. Ciarlet, S. E. Crawford, E. Cheng, et al., "VLA-2 ( $\alpha 2 \beta 1)$ integrin promotes rotavirus entry into cells but is not necessary for rotavirus attachment," Journal of Virology, vol. 76, no. 3, pp. 1109-1123, 2002.

[23] M. K. Estes, D. Y. Graham, and B. B. Mason, "Proteolytic enhancement of rotavirus infectivity: molecular mechanisms," Journal of Virology, vol. 39, no. 3, pp. 879-888, 1981.

[24] K. L. Graham, Y. Takada, and B. S. Coulson, "Rotavirus spike protein VP5* binds $\alpha 2 \beta 1$ integrin on the cell surface and competes with virus for cell binding and infectivity," Journal of General Virology, vol. 87, no. 5, pp. 1275-1283, 2006.

[25] C. Istrate, I. Douagi, A. Charpilienne, et al., "Bone marrow dendritic cells internalize live RF-81 bovine rotavirus and rotavirus-like particles (RF 2/6-GFP-VLP and RF $8 * 2 / 6 / 7$ VLP) but are only activated by live bovine rotavirus," Scandinavian Journal of Immunology, vol. 65, no. 6, pp. 494-502, 2007.

[26] C. Guerin-Danan, J. C. Meslin, F. Lambre, et al., "Development of a heterologous model in germfree suckling rats for studies of rotavirus diarrhea," Journal of Virology, vol. 72, no. 11, pp. 9298-9302, 1998. 


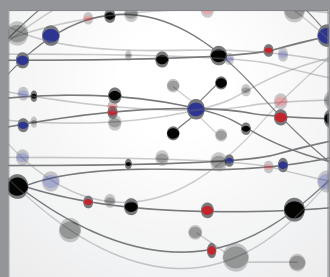

The Scientific World Journal
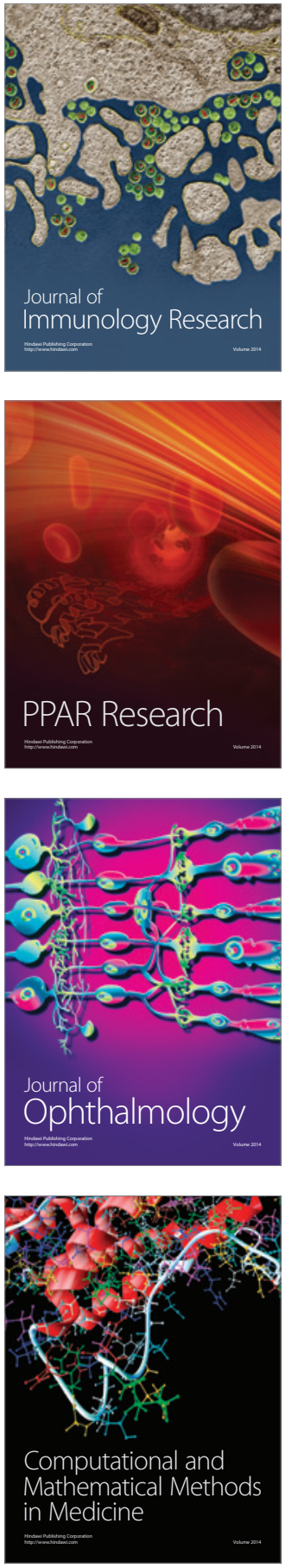

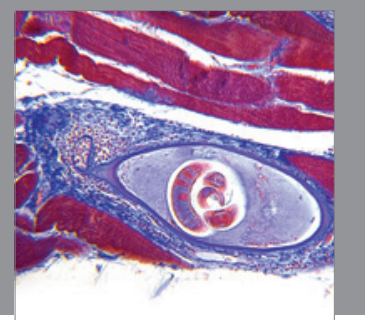

Gastroenterology

Research and Practice
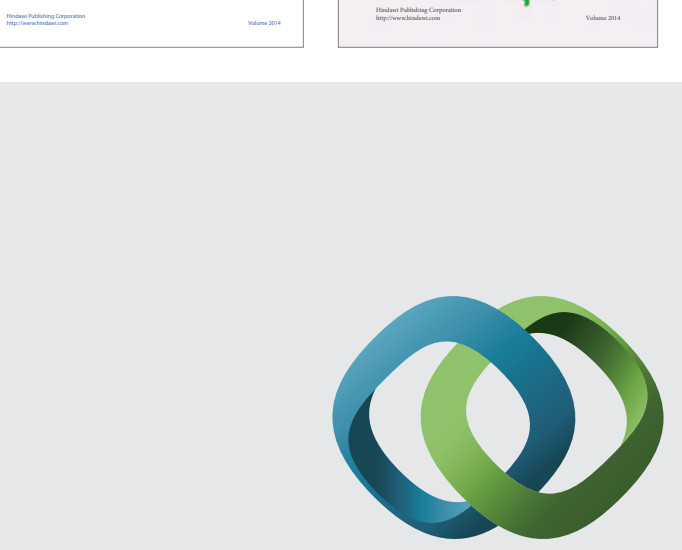

\section{Hindawi}

Submit your manuscripts at

http://www.hindawi.com
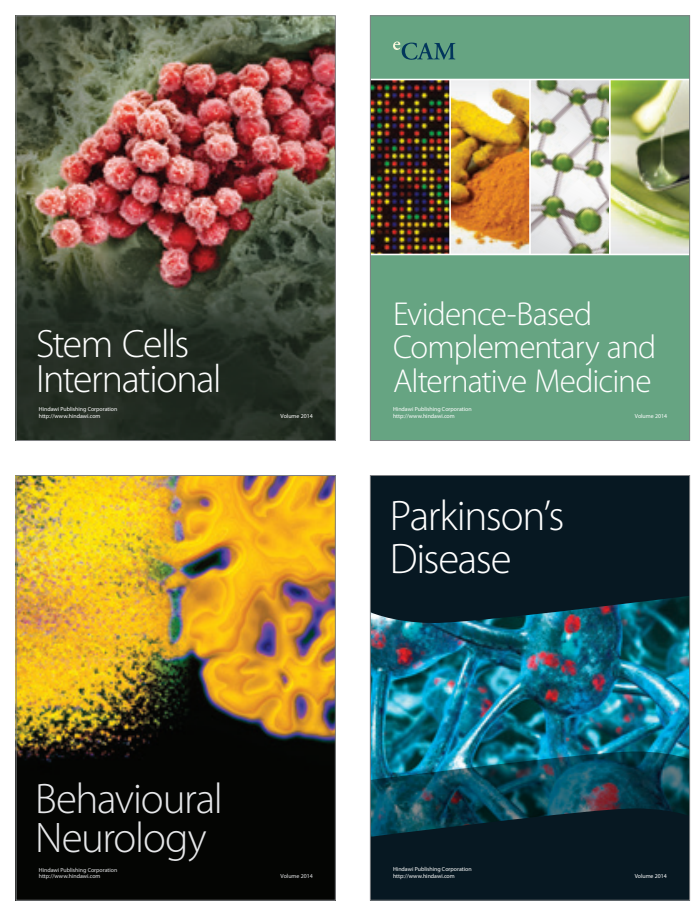

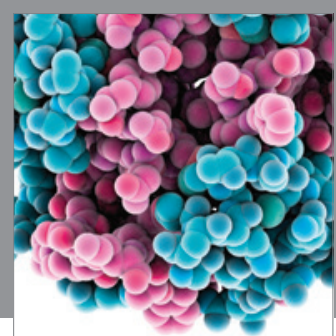

Journal of
Diabetes Research

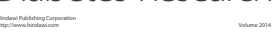

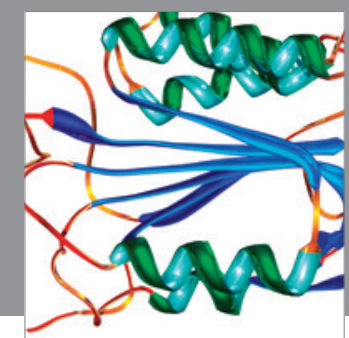

Disease Markers
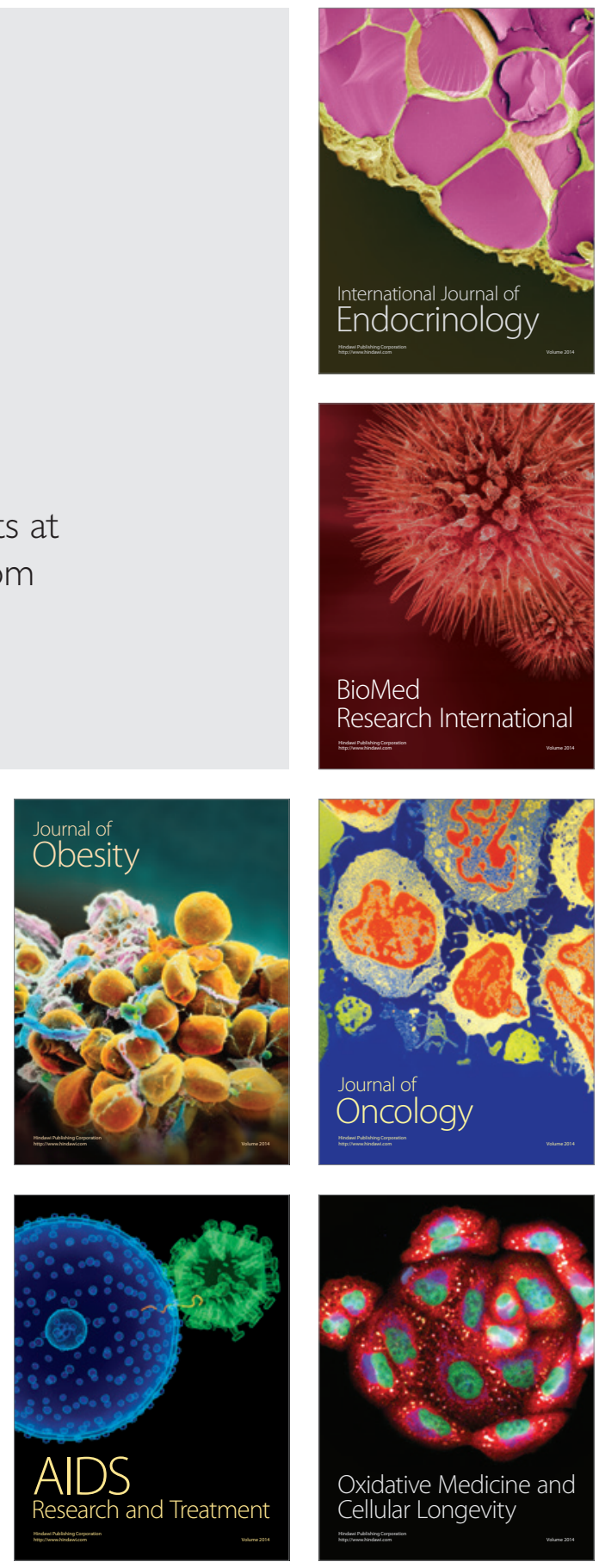